\title{
Quantum Monte Carlo Calculations for Light Nuclei
}

\author{
R. B. Wiringa
}

Physics Division, Argonne National Laboratory, Argonne, IL 60439 , USA

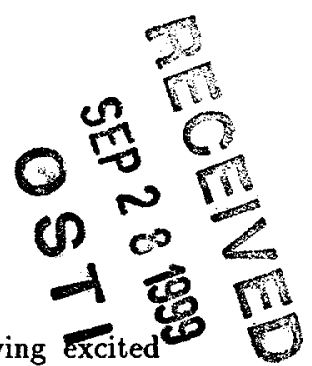

Abstract. Quantum Monte Carlo calculations of ground and low-lying excited states for nuclei with $A \leq 8$ are made using a realistic Hamiltonian that fits $N N$ scattering data. Results for more than 40 different $\left(J^{\pi}, T\right)$ states, plus isobaric analogs, are obtained and the known excitation spectra are reproduced reasonably well. Various density and momentum distributions and electromagnetic form factors and moments have also been computed. These are the first microscopic calculations that directly produce nuclear shell structure from realistic $N N$ interactions.

\section{Introduction}

Tremendous progress has been made in this decade in the characterization of nuclear forces, with sophisticated new partial-wave analyses and a number of $N N$ potential models that fit data with a $\chi^{2} /$ point near unity [1]. Concurrently, several essentially exact methods have been developed for the study of few-nucleon systems with such realistic two- and three-nucleon interactions [2]. The success in describing both bound and scattering state data is profound. New developments in quantum Monte Carlo methods are now making the light $\mathrm{p}$-shell nuclei accessible at the same level of accuracy $[3,4]$. This is opening new possibilities to test our $N N$ force models in P-waves and models of three-nucleon forces in $T=\frac{3}{2}$ states.

Here I summarize recent quantum Monte Carlo calculations $[3,4]$ of ground and low-lying excited states for nuclei with $A \leq 8$. These have been made using a Hamiltonian containing the Argonne $v_{18}$ two-nucleon and Urbana IX three-nucleon potentials. The first step in these calculations is the construction of a variational wave function, $\Psi_{V}\left(J^{\pi}, T\right)$, using products of twoand three-body correlation operators acting on a fully antisymmetrized set of one-body p-shell basis states coupled to the specified quantum numbers. Metropolis Monte Carlo integration is used to evaluate $\left\langle\Psi_{V}|H| \Psi_{V}\right\rangle$ and diagonalize in the one-body p-shell basis, giving an upper bound to the specific state. The $\Psi_{V}$ is then used as input to the Green's function Monte Carlo algorithm, which projects out excited state contamination in the trial wave function by the Euclidean propagation $\Psi(\tau)=\exp \left[-\left(H-E_{0}\right) \tau\right] \Psi_{V}$; in the limit $\tau \rightarrow \infty$, this yields the exact $\Psi_{0}$. In practice propagation to $\tau=0.06-$ $0.10 \mathrm{MeV}^{-1}$ gives a significantly improved upper bound compared to the 


\section{DISCLAIMER}

This report was prepared as an account of work sponsored by an agency of the United States Government. Neither the United States Government nor any agency thereof, nor any of their employees, make any warranty, express or implied, or assumes any legal liability or responsibility for the accuracy, completeness, or usefulness of any information, apparatus, product, or process disclosed, or represents that its use would not infringe privately owned rights. Reference herein to any specific commercial product, process, or service by trade name, trademark, manufacturer, or otherwise does not necessarily constitute or imply its endorsement, recommendation, or favoring by the United States Government or any agency thereof. The views and opinions of authors expressed herein do not necessarily state or reflect those of the United States Government or any agency thereof. 


\title{
DISCLAIMER
}

\author{
Portions of this document may be illegible \\ in electronic image products. Images are \\ produced from the best available original \\ document.
}


variational result, which we estimate to be within $\sim 1 \%$ of the exact binding energy for this Hamiltonian.

We obtain ground states of $\mathrm{Li}$ isotopes that are stable against breakup into subclusters, but somewhat underbound compared to experiment. Many other states in the $A=6-8$ nuclei are also calculated, reproducing the known excitation spectra of these nuclei reasonably well while predicting a number of additional excited states in the He isotopes. Energy differences between isobaric analogs are also computed to study the charge-independence-breaking of the strong and electromagnetic forces. Density and momentum distributions are generated, along with electromagnetic moments and form factors. These are the first microscopic calculations that directly produce nuclear shell structure from realistic interactions that fit $N N$ scattering data.

\section{Hamiltonian}

Our Hamiltonian includes nonrelativistic kinetic energy, the Argonne $v_{18}$ twonucleon potential [5] and the Urbana IX three-nucleon potential [3]:

$$
H=\sum_{i} K_{i}+\sum_{i<j} v_{i j}+\sum_{i<j<k} V_{i j k} .
$$

The kinetic energy operator has charge-independent (CI) and charge-symmetrybreaking (CSB) components, the latter due to the difference in proton and neutron masses. The Argonne $v_{18}$ potential is written as a sum of electromagnetic and one-pion-exchange terms and a shorter-range phenomenological part,

$$
v_{i j}=v_{i j}^{\gamma}+v_{i j}^{\pi}+v_{i j}^{R} .
$$

The electromagnetic terms include one- and two-photon-exchange Coulomb interaction, vacuum polarization, Darwin-Foldy, and magnetic moment terms (which have spin-spin, tensor, and spin-orbit structure) with appropriate proton and neutron form factors. The one-pion-exchange part of the potential includes the charge-dependent (CD) terms due to the difference in neutral and charged pion masses. The remaining terms are of intermediate (twopion-exchange) and short range, with some 40 adjustable parameters. The potential was fit directly to the Nijmegen $N N$ scattering data base $[6,7]$ containing $1787 p p$ and $2514 n p$ data in the range $0-350 \mathrm{MeV}$, with a $\chi^{2}$ per datum of 1.09 . It was also fit to the $n n$ scattering length and the deuteron binding energy.

The one-pion-exchange and the remaining phenomenological part of the potential can be written as a sum of eighteen operators,

$$
v_{i j}^{\pi}+v_{i j}^{R}=\sum_{p=1,18} v_{p}\left(r_{i j}\right) O_{i j}^{p} .
$$

The first fourteen are CI operators, 


$$
O_{i j}^{p=1,14}=\left[1,\left(\sigma_{i} \cdot \sigma_{j}\right), S_{i j},(\mathbf{L} \cdot \mathbf{S}), \mathbf{L}^{2}, \mathbf{L}^{2}\left(\sigma_{i} \cdot \sigma_{j}\right),(\mathbf{L} \cdot \mathbf{S})^{2}\right] \otimes\left[1,\left(\tau_{i} \cdot \tau_{j}\right)\right],
$$

while the last four,

$$
O_{i j}^{p=15,18}=\left[1,\left(\sigma_{i} \cdot \sigma_{j}\right), S_{i j}\right] \otimes T_{i j}, \text { and }\left(\tau_{z i}+\tau_{z j}\right),
$$

are the CD and CSB terms.

The Urbana series of three-nucleon potentials is written as a sum of twopion-exchange and shorter-range phenomenological terms,

$$
V_{i j k}=V_{i j k}^{2 \pi}+V_{i j k}^{R} \text {. }
$$

The $V_{i j k}^{2 \pi}$ can be expressed as a sum of anticommutator and commutator terms built from one-pion-exchange elements taken from the $N N$ force, as in the original Fujita-Miyazawa model [8]. The $V_{i j k}^{R}$ has no spin-isospin dependence in the present model, although in principle it could be quite complicated [9]; presumably a part of it is due to relativistic effects $[10,11]$. The strengths of the two components have been determined by fitting the saturation density of nuclear matter and the binding energy of ${ }^{3} \mathrm{H}$ in conjunction with the Argonne $v_{18}$ interaction.

\section{Variational Monte Carlo}

The variational method can be used to obtain approximate solutions to the many-body Schrödinger equation, $H \Psi=E \Psi$, for a wide range of nuclear systems, including few-body nuclei, light closed shell nuclei, nuclear matter, and neutron stars [12]. A suitably parameterized wave function, $\Psi_{V}$, is used to calculate an upper bound to the exact ground-state energy,

$$
E_{V}=\frac{\left\langle\Psi_{V}|H| \Psi_{V}\right\rangle}{\left\langle\Psi_{V} \mid \Psi_{V}\right\rangle} \geq E_{0}
$$

The parameters in $\Psi_{V}$ are varied to minimize $E_{V}$, and the lowest value is taken as the approximate ground-state energy. Upper bounds to excited states are also obtainable, if they have different quantum numbers from the ground state, or from small-basis diagonalizations if they have the same quantum numbers. The corresponding $\Psi_{V}$ can then be used to calculate other properties, such as particle density or electromagnetic moments, or they can be used as starting points for Green's function Monte Carlo calculations.

Our best variational wave function has the form [13]

$$
\left|\Psi_{V}\right\rangle=\left[1+\sum_{i<j<k}\left(U_{i j k}+U_{i j k}^{T N I}\right)+\sum_{i<j} U_{i j}^{L S}\right]\left|\Psi_{P}\right\rangle,
$$

where the pair wave function, $\Psi_{P}$, is given by 


$$
\left|\Psi_{P}\right\rangle=\left[\mathcal{S} \prod_{i<j}\left(1+U_{i j}\right)\right]\left|\Psi_{J}\right\rangle
$$

The $U_{i j}, U_{i j}^{L S}, U_{i j k}$, and $U_{i j k}^{T N I}$ are noncommuting two- and three-nucleon correlation operators, and $\mathcal{S}$ is a symmetrization operator. The form of the antisymmetric Jastrow wave function, $\Psi_{J}$, depends on the nuclear state under investigation. For the s-shell nuclei we use the simple form

$$
\left|\Psi_{J}\right\rangle=\left[\prod_{i<j<k} f_{i j k}^{c}\left(\mathbf{r}_{i k}, \mathbf{r}_{j k}\right) \prod_{i<j} f_{c}\left(r_{i j}\right)\right]\left|\Phi_{A}\left(J M T T_{3}\right)\right\rangle .
$$

Here $f_{c}\left(r_{i j}\right)$ and $f_{i j k}^{c}$ are central (operator-independent) two- and three-body correlation functions and $\Phi_{A}$ is an antisymmetrized spin-isospin array, e.g.,

$$
\left|\Phi_{4}(0000)\right\rangle=\mathcal{A} \mid p \nmid p\lfloor n \uparrow n \downarrow\rangle .
$$

The two-body correlation operator $U_{i j}$ is a sum of spin, isospin, and tensor terms:

$$
U_{i j}=\sum_{p=2,6}\left[\prod_{k \neq i, j} f_{i j k}^{p}\left(\mathbf{r}_{i k}, \mathbf{r}_{j k}\right)\right] u_{p}\left(r_{i j}\right) O_{i j}^{p},
$$

with a similar structure for $U_{i j}^{L S}$; these correlations are induced by $v_{i j}$. The equations used to generate the functions $f_{c}\left(r_{i j}\right)$ and $u_{p}\left(r_{i j}\right)$ contain a number of variational parameters to be determined by minimizing the energy [14]. The $f_{i j k}^{c}, f_{i j k}^{p}$, and $U_{i j k}$ are three-nucleon correlations also induced by $v_{i j}$ [13], while the $U_{i j k}^{T N I}$ is a three-body correlation induced by the three-nucleon interaction. A somewhat simpler wave function to evaluate is given by:

$$
\left|\Psi_{T}\right\rangle=\left[1+\sum_{i<j<k} \tilde{U}_{i j k}^{T N I}\right]\left|\Psi_{P}\right\rangle
$$

where $\tilde{U}_{i j k}^{T N I}$ is a slightly truncated TNI correlation. The $\Psi_{T}$ gives about $1-2$ $\mathrm{MeV}$ less binding than the full $\Psi_{V}$, but costs less than half as much to compute, making it a more efficient starting point for the GFMC calculation [4].

The Jastrow wave function for $A=6$ nuclei is more complicated, as two nucleons must be placed in the p-shell. We use $L S$ coupling to obtain the desired $J M$ value of a given state, as suggested in shell-model studies of p-shell nuclei [15]. Different possible $L S$ combinations lead to multiple components in the Jastrow wave function. We also allow for the possibility that the central correlations $f_{c}\left(r_{i j}\right)$ could depend upon the shells ( $\mathrm{s}$ or $\mathrm{p}$ ) occupied by the particles and on the $L S$ coupling: 


$$
\begin{gathered}
\left|\Psi_{J}\right\rangle=\mathcal{A}\left\{\prod_{i<j<k \leq 4} f_{i j k}^{c} \prod_{i<j \leq 4} f_{s s}\left(r_{i j}\right) \prod_{k \leq 4} f_{s p}\left(r_{k 5}\right) f_{s p}\left(r_{k 6}\right)\right. \\
\left.\left.\sum_{L S}\left(\beta_{L S} f_{p p}^{L S}\left(r_{56}\right) \mid \Phi_{6}\left(L S J M T T_{3}\right)_{1234: 56}\right)\right)\right\} .
\end{gathered}
$$

The operator $\mathcal{A}$ indicates an antisymmetric sum over all possible partitions of the six particles into $4 \mathrm{~s}$-shell and $2 \mathrm{p}$-shell ones. The central correlation $f_{s s}(r)$ is the $f_{c}(r)$ from the ${ }^{4} \mathrm{He}$ wave function, while the $f_{s p}(r)$ and $f_{p p}(r)$ have a similar shape with long-range tails added.

The $L S$ components of the single-particle wave function are given by:

$$
\begin{aligned}
& \left|\Phi_{6}\left(L S J M T T_{3}\right)_{1234: 56}\right\rangle=\mid \Phi_{4}(0000)_{1234} \phi_{p}^{L S}\left(R_{\alpha 5}\right) \phi_{p}^{L S}\left(R_{\alpha 6}\right) \\
& \left\{\left[Y_{1 m_{l}}\left(\Omega_{\alpha 5}\right) Y_{1 m_{l}^{\prime}}\left(\Omega_{\alpha 6}\right)\right]_{L M_{L}} \times\left[\chi_{5}\left(\frac{1}{2} m_{s}\right) \chi_{6}\left(\frac{1}{2} m_{s}^{\prime}\right)\right]_{S M_{S}}\right\}_{J M} \\
& \left.\times\left[\nu_{5}\left(\frac{1}{2} t_{3}\right) \nu_{6}\left(\frac{1}{2} t_{3}^{\prime}\right)\right]_{T T_{3}}\right\rangle .
\end{aligned}
$$

The $\phi_{p}^{L S}\left(R_{\alpha k}\right)$ are p-wave solutions of a particle in an effective $\alpha-N$ potential and are functions of the distance between the center of mass of the $\alpha$ core and nucleon $k$; they may be different for different $L S$ components.

For $A=7,8$ nuclei, the generalization of $\left|\Psi_{J}\right\rangle$ is straightforward, except that with three or more $\mathrm{p}$-shell particles there are multiple ways of coupling to obtain the orbital angular momentum, $L$, and spin, $S$, and thus the total $\left(J^{\pi}, T\right)$ of the system. The spatial permutation symmetry, denoted by the Young pattern $[n]$, is used to enumerate the different possible terms; the different possible contributions to $A=6-8$ nuclei are given in Ref. [16]. After other parameters in the trial function have been optimized, we make a series of calculations in which the $\beta_{L S[n]}$ of Eq.(14) may be different in the leftand right-hand-side wave functions to obtain the diagonal and off-diagonal matrix elements of the Hamiltonian and the corresponding normalizations and overlaps. We diagonalize the resulting $\mathrm{N} \times \mathrm{N}$ matrices to find the $\beta_{L S[n]}$ eigenvectors, using generalized eigenvalue routines because the correlated $\Psi_{V}$ are not orthogonal. This allows us to project out not only the ground states, but excited states of the same $\left(J^{\pi}, T\right)$ quantum numbers. For example, the ground states of ${ }^{6} \mathrm{Li},{ }^{7} \mathrm{Li}$, and ${ }^{8} \mathrm{Li}$ are obtained from $3 \times 3,5 \times 5$, and $7 \times 7$ diagonalizations, respectively.

The energy expectation value of Eq.(7) is evaluated using Monte Carlo integration. A detailed technical description of the methods used here can be found in Refs. [14, 17]. Monte Carlo sampling is done both in configuration space and in the order of operators in the symmetrized product of Eq.(9) by following a Metropolis random walk. The expectation value for an operator $O$ is computed with the expression

$$
\langle O\rangle=\frac{\sum_{p, q} \int d \mathbf{R}\left[\Psi_{p}^{\dagger}(\mathbf{R}) O \Psi_{q}(\mathbf{R}) / W_{p q}(\mathbf{R})\right] W_{p q}(\mathbf{R})}{\sum_{p, q} \int d \mathbf{R}\left[\Psi_{p}^{\dagger}(\mathbf{R}) \Psi_{q}(\mathbf{R}) / W_{p q}(\mathbf{R})\right] W_{p q}(\mathbf{R})},
$$


where samples are drawn from a probability distribution, $W_{p q}(\mathbf{R})$, which is proportional to $\left|\Psi_{P}\right|^{2}$. Expectation values have a statistical error given by the standard deviation, $\sigma$.

A key feature of the nuclear Monte Carlo calculations is the spin-isospin structure of the wave function $\Psi$ :

$$
\Psi(\mathbf{R})=\sum_{\alpha} \psi_{\alpha}(\mathbf{R})|\alpha\rangle
$$

There are $2^{A} \times I(A, T)$ components $\alpha$ which specify the third components of spin and the possible isospin states. This gives arrays with $32,320,1792$, and 7168 elements in $\Psi$ for ${ }^{4} \mathrm{He},{ }^{6} \mathrm{Li},{ }^{7} \mathrm{Li}$, and ${ }^{8} \mathrm{Li}$, respectively. The spin, isospin, and tensor operators $O_{i j}^{p=2,6}$ contained in the two-body correlation operator $U_{i j}$, and in the Hamiltonian are sparse matrices in this basis. The rapid growth in the size $\Psi$ as $A$ increases is the chief limitation on extending the current method to larger nuclei.

A major problem arises in minimizing the variational energy for $\mathrm{p}$-shell nuclei using the above wave functions: there is no variational minimum that gives reasonable rms radii. For example, the variational energy for ${ }^{6} \mathrm{Li}$ is slightly more bound than for ${ }^{4} \mathrm{He}$, but is not more bound than for separated ${ }^{4} \mathrm{He}$ and ${ }^{2} \mathrm{H}$ nuclei, so the wave function is not stable against breakup into $\alpha+d$ subclusters. Consequently, the energy can be lowered toward the sum of ${ }^{4} \mathrm{He}$ and ${ }^{2} \mathrm{H}$ energies by making the wave function more and more diffuse. Such a diffuse wave function would not be useful for computing other nuclear properties, or as a starting point for the GFMC calculation, so we constrain our search for variational parameters by requiring the resulting point proton rms radius, $r_{p}$, to be close to the experimental values for ${ }^{6} \mathrm{Li}$ and ${ }^{7} \mathrm{Li}$ ground states. For other $A=6-8$ ground states, and all the excited states, we construct trial functions by making minimal changes to the ${ }^{6} \mathrm{Li}$ and ${ }^{7} \mathrm{Li}$ wave functions, with the added requirement that excited states should not have smaller radii than the ground states. The final step is always the diagonalization of the Hamiltonian in the $\beta_{L S[n]}$ mixing parameters.

\section{Green's Function Monte Carlo}

The GFMC method [18] projects out the exact lowest-energy state, $\Psi_{0}$, for a given set of quantum numbers, using $\Psi_{0}=\lim _{\tau \rightarrow \infty} \exp \left[-\left(H-E_{0}\right) \tau\right] \Psi_{T}$, where $\Psi_{T}$ is an initial trial function. The eigenvalue $E_{0}$ is calculated exactly while other expectation values are generally calculated neglecting terms of order $\left|\Psi_{0}-\Psi_{T}\right|^{2}$ and higher [4]. In contrast, the error in the variational energy, $E_{V}$, is of order $\left|\Psi_{0}-\Psi_{T}\right|^{2}$, and other expectation values calculated with $\Psi_{T}$ have errors of order $\left|\Psi_{0}-\Psi_{T}\right|$.

We use the $\Psi_{T}$ of $\mathrm{Eq} .(13)$ and define the propagated wave function $\Psi(\tau)$

$$
\Psi(\tau)=e^{-\left(H-E_{0}\right) r} \Psi_{T}=\left[e^{-\left(H-E_{0}\right) \Delta \tau}\right]^{n} \Psi_{T}
$$


where we have introduced a small time step, $\tau=n \Delta \tau$; obviously $\Psi(\tau=0)=$ $\Psi_{T}$ and $\Psi(\tau \rightarrow \infty)=\Psi_{0}$. The $\Psi(\tau)$ is represented by a vector function of $\mathbf{R}$ using Eq.(17), and the Green's function, $G_{\alpha \beta}\left(\mathbf{R}, \mathbf{R}^{\prime}\right)$ is a matrix function of $\mathbf{R}$ and $\mathbf{R}^{\prime}$ in spin-isospin space, defined as

$$
G_{\alpha \beta}\left(\mathbf{R}, \mathbf{R}^{\prime}\right)=\left\langle\mathbf{R}, \alpha\left|e^{-\left(H-E_{0}\right) \Delta \tau}\right| \mathbf{R}^{\prime}, \beta\right\rangle .
$$

It is calculated with leading errors of order $(\Delta \tau)^{3}$. Omitting spin-isospin indices for brevity, $\Psi\left(\mathbf{R}_{n}, \tau\right)$ is given by

$$
\Psi\left(\mathbf{R}_{n}, \tau\right)=\int G\left(\mathbf{R}_{n}, \mathbf{R}_{n-1}\right) \cdots G\left(\mathbf{R}_{1}, \mathbf{R}_{0}\right) \Psi_{T}\left(\mathbf{R}_{0}\right) d \mathbf{R}_{n-1} \cdots \mathbf{R}_{1} d \mathbf{R}_{0} .
$$

with the integral being evaluated stochastically.

The short-time propagator should allow as large a time step $\Delta \tau$ as possible, because the total computational time for propagation is proportional to $1 / \Delta \tau$. An efficient propagator can be constructed from a symmetrized product of exact two-body propagators, $g_{i j}$, as

$$
G_{\alpha \beta}\left(\mathbf{R}, \mathbf{R}^{\prime}\right)=G_{0}\left(\mathbf{R}, \mathbf{R}^{\prime}\right)\left\langle\alpha\left|\left[\mathcal{S} \prod_{i<j} \frac{g_{i j}\left(\mathbf{r}_{i j}, \mathbf{r}_{i j}^{\prime}\right)}{g_{0, i j}\left(\mathbf{r}_{i j}, \mathbf{r}_{i j}^{\prime}\right)}\right]\right| \beta\right\rangle,
$$

with $g_{0, i j}$ as the free two-body propagator. It has errors of order $(\Delta \tau)^{3}$, but they are from commutators of terms like $v_{i j} T v_{i k}(\Delta \tau)^{3}$ which become large only when all three particles $i j k$ are close. Thus a fairly large time step $\Delta \tau \sim 0.5 \mathrm{GeV}^{-1}$ can be used [4]. The complete propagator includes the three-body forces and the $E_{0}$ in Eq.(19).

Quantities of interest are evaluated in terms of a mixed expectation value:

$$
\begin{aligned}
\langle O\rangle_{\text {Mixed }} & =\frac{\left\langle\Psi_{T}|O| \Psi(\tau)\right\rangle}{\left\langle\Psi_{T} \mid \Psi(\tau)\right\rangle} \\
& =\frac{\int d \mathbf{P}_{n} \Psi_{T}^{\dagger}\left(\mathbf{R}_{n}\right) O G\left(\mathbf{R}_{n}, \mathbf{R}_{n-1}\right) \cdots G\left(\mathbf{R}_{1}, \mathbf{R}_{0}\right) \Psi_{T}\left(\mathbf{R}_{0}\right)}{\int d \mathbf{P}_{n} \Psi_{T}^{\dagger}\left(\mathbf{R}_{n}\right) G\left(\mathbf{R}_{n}, \mathbf{R}_{n-1}\right) \cdots G\left(\mathbf{R}_{1}, \mathbf{R}_{0}\right) \Psi_{T}\left(\mathbf{R}_{0}\right)}
\end{aligned}
$$

where $\mathbf{P}_{n}=\mathbf{R}_{0}, \mathbf{R}_{1}, \cdots, \mathbf{R}_{n}$ denotes the 'path', and $d \mathbf{P}_{n}=d \mathbf{R}_{0} d \mathbf{R}_{1} \cdots d \mathbf{R}_{n}$ with the integral over the paths being carried out stochastically. Assuming $\Psi(\tau)=\Psi_{T}+\delta \Psi(\tau)$, we obtain the approximate expression

$$
\langle O(\tau)\rangle=\frac{\langle\Psi(\tau)|O| \Psi(\tau)\rangle}{\langle\Psi(\tau) \mid \Psi(\tau)\rangle} \approx\langle O(\tau)\rangle_{\text {Mixed }}+\left[\langle O(\tau)\rangle_{\text {Mixed }}-\langle O\rangle_{T}\right]
$$

where $\langle O\rangle_{T}$ is the variational expectation value. A special case is the expectation value of the Hamiltonian. The $\langle H(\tau)\rangle_{\text {Mixed }}$ can be re-expressed as [19]

$$
\langle H(\tau)\rangle_{\text {Mixed }}=\frac{\left\langle\Psi_{T}\left|e^{-\left(H-E_{0}\right) \tau / 2} H e^{-\left(H-E_{0}\right) \tau / 2}\right| \Psi_{T}\right\rangle}{\left\langle\Psi_{T}\left|e^{-\left(H-E_{0}\right) \tau / 2} e^{-\left(H-E_{0}\right) \tau / 2}\right| \Psi_{T}\right\rangle} \geq E_{0}
$$


since the propagator $\exp \left[-\left(H-E_{0}\right) \tau\right]$ commutes with the Hamiltonian. Thus $\langle H(\tau)\rangle_{\text {Mixed }}$ approaches $E_{0}$ in the limit $\tau \rightarrow \infty$, and the expectation value obeys the variational principle for all $\tau$.

Direct GFMC calculations with the full interaction (in particular spindependent terms which involve the square of the momentum operator) have very large statistical errors. Hence we construct the GFMC propagator with a simpler isoscalar interaction, $H^{\prime}$, with a $v_{i j}^{\prime}$ that has only eight operator terms, $\left[1,\left(\sigma_{i} \cdot \sigma_{j}\right), S_{i j},(\mathbf{L} \cdot \mathbf{S})\right] \otimes\left[1,\left(\tau_{i} \cdot \tau_{j}\right)\right]$, chosen such that it equals the isoscalar part of the full interaction in all $\mathrm{S}$ - and $\mathrm{P}$-waves and in the deuteron. The $v_{i j}^{\prime}$ is a little more attractive than $v_{i j}$, so we also use a $V_{i j k}^{\prime}$ adjusted so that $\left\langle H^{\prime}\right\rangle \approx\langle H\rangle$. This ensures the GFMC algorithm will not propagate to excessively large densities due to overbinding. Consequently, the upper bound property applies to $\left\langle H^{\prime}(\tau)\right\}$, and $\left\langle H-H^{\prime}\right\rangle$ must be evaluated perturbatively.

Another complication that arises in the GFMC algorithm is the 'fermion sign problem'. Wave functions of simple Fermi systems have domains of positive and negative signs separated by nodal surfaces. When the path $\mathbf{P}$ crosses a nodal surface, the variance of the mixed expectation value starts to increase. This seldom happens for small time steps, but as $\tau$ increases, this noise builds up and it effectively limits the value of $\tau$ to $0.06 \mathrm{MeV}^{-1}$ for most of the nuclei studied here. The problem is exacerbated if one has a poor starting trial function $\Psi_{T}$.

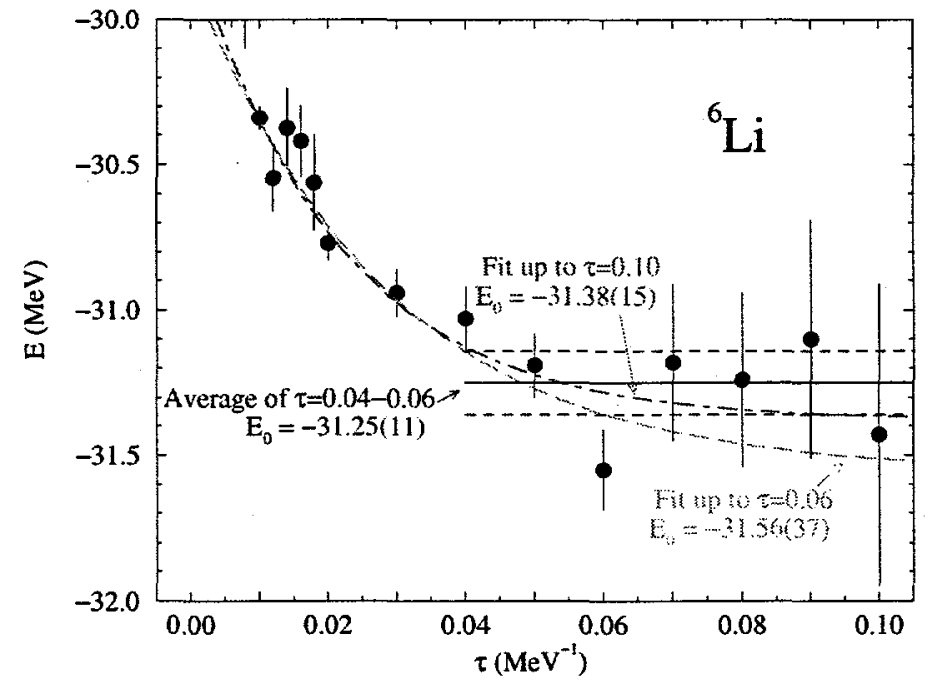

Fig. 1. GFMC transient energy for ${ }^{6} \mathrm{Li}$

The progress of a GFMC calculation is shown in Fig. 1, where $E(\tau)$ for ${ }^{6} \mathrm{Li}$ is plotted. The GFMC energies are shown by points with error bars; it 
can be seen that the error grows with increasing $\tau$. Most of our calculations are made to $\tau=0.06 \mathrm{MeV}^{-1}$, but in this case they were pushed to $\tau=$ $0.10 \mathrm{MeV}^{-1}$. The curves shown are attempts to extrapolate $E(\tau)$ with a sum of exponentially decaying excited states; we conclude it is safest to simply take the average of the last few points as the GFMC energy. We believe these averages are reliable upper bounds to the eigenenergies of $H$, and are probably within $\sim 0.3(\sim 0.5) \mathrm{MeV}$ of the exact energy for $A=6(A=7)$ nuclei [4].

The calculations described here are computationally intense, and would not have been possible without the advent of parallel supercomputers. Our initial studies [3] of ${ }^{6} \mathrm{Li}$ required $\sim 2,000$ node hours on an IBM SP1 to propagate 10,000 configurations to $\tau=0.06 \mathrm{MeV}^{-1}$. Today, with improvements in the algorithm and after much effort to optimize the computer codes, the same calculation requires only 6 node hours on a third generation IBM SP. However, a present calculation for ${ }^{8} \mathrm{He}$, requiring 50,000 configurations to get a reasonable error bar, takes 600 node hours, so forefront computer resources remain essential for this program.

\section{$5 \quad$ Energy Results}

The chief results of this work are the ground and excited state energies shown in Figs. 2 and 3 . The results for $A=6,7$ have been reported previously [4]; the results for $A=8$ are preliminary. The VMC calculations using $\Psi_{V}$ are $2 \%$ above the GFMC ground state results for $A=3,4$ nuclei, the latter having been fit to the experimental energies by construction of $V_{i j k}$. However, the variational calculations get progressively worse for $A=6-8$, being $10-12 \%$ above the final GFMC results, for nuclei with $N \sim Z$. In absolute terms, $\Psi_{V}$ misses an extra $1.5 \mathrm{MeV}$ of binding for each p-shell nucleon that is added. Clearly, there is some significant feature of light p-shell nuclei that we do not yet know how to model in our trial functions.

We also observe that the GFMC results are underbound compared to experiment. The $\mathrm{Li}$ isotopes are stable against breakup into subclusters, but progressively more underbound as $A$ increases. This is probably a failure of our Hamiltonian. There is also an isospin problem with our interaction, in that the He isotopes are even further off from the experimental values, and in fact do not show stability against breakup into subclusters.

A detailed breakdown of the GFMC interaction energy into kinetic, twoand three-nucleon interactions is given in Ref. [4]. There is a big cancellation between the kinetic and two-body potential terms. Consequently, while $V_{i j k}$ is less than $5 \%$ of $v_{i j}$ in magnitude, it contributes up to $25 \%$ of the net binding. Among the subcomponents of $v_{i j}$, the one-pion exchange dominates, being $70-75 \%$ of the total $v_{i j}$ for $N=Z$ nuclei. Similarly, the two-pion exchange is the dominant component of $V_{i j k}$, while the shorter-range $V_{i j k}^{R}$ is much larger than the discrepancy between the total GFMC energy and 


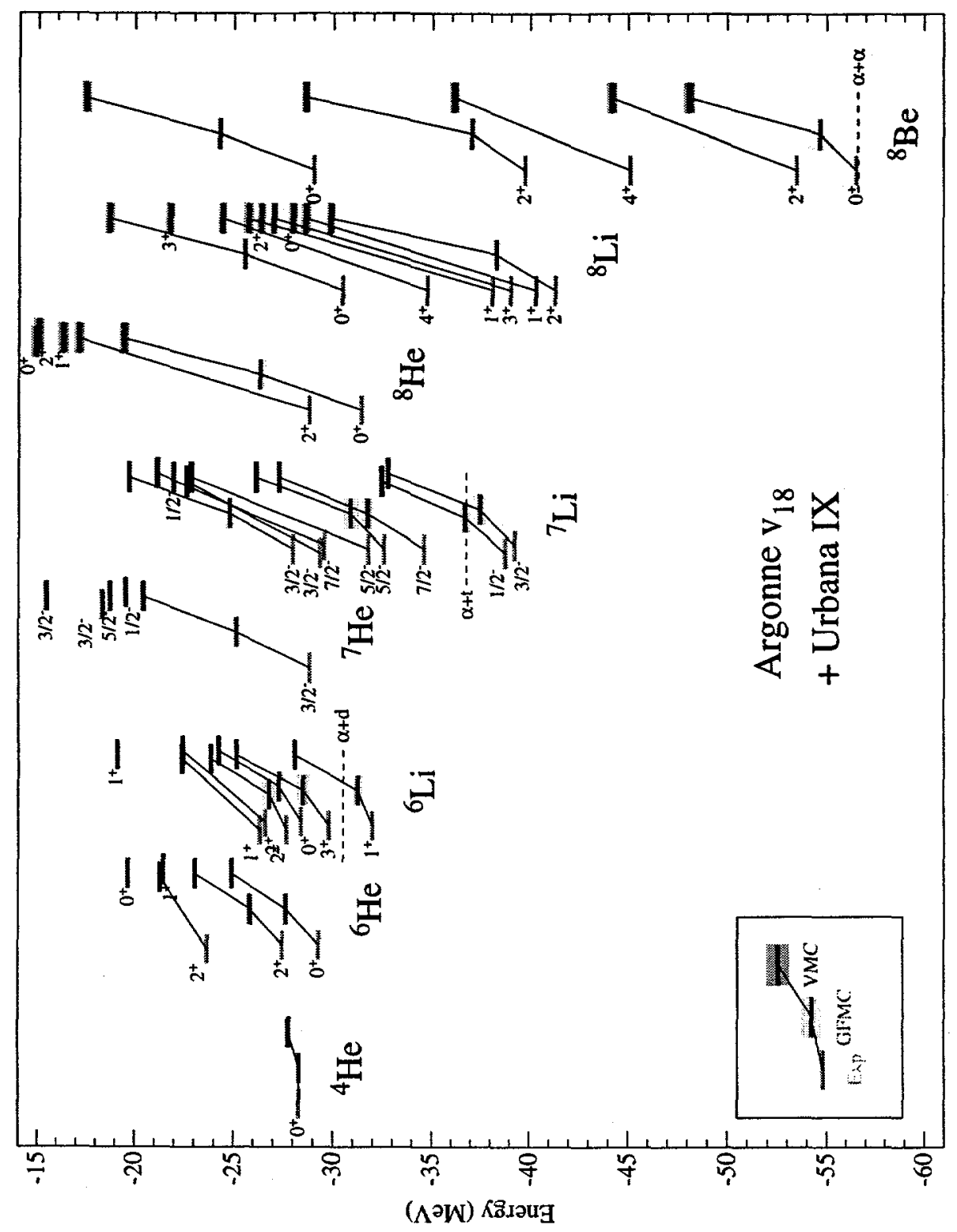

Fig. 2. Experimental and calculated energy spectrum 


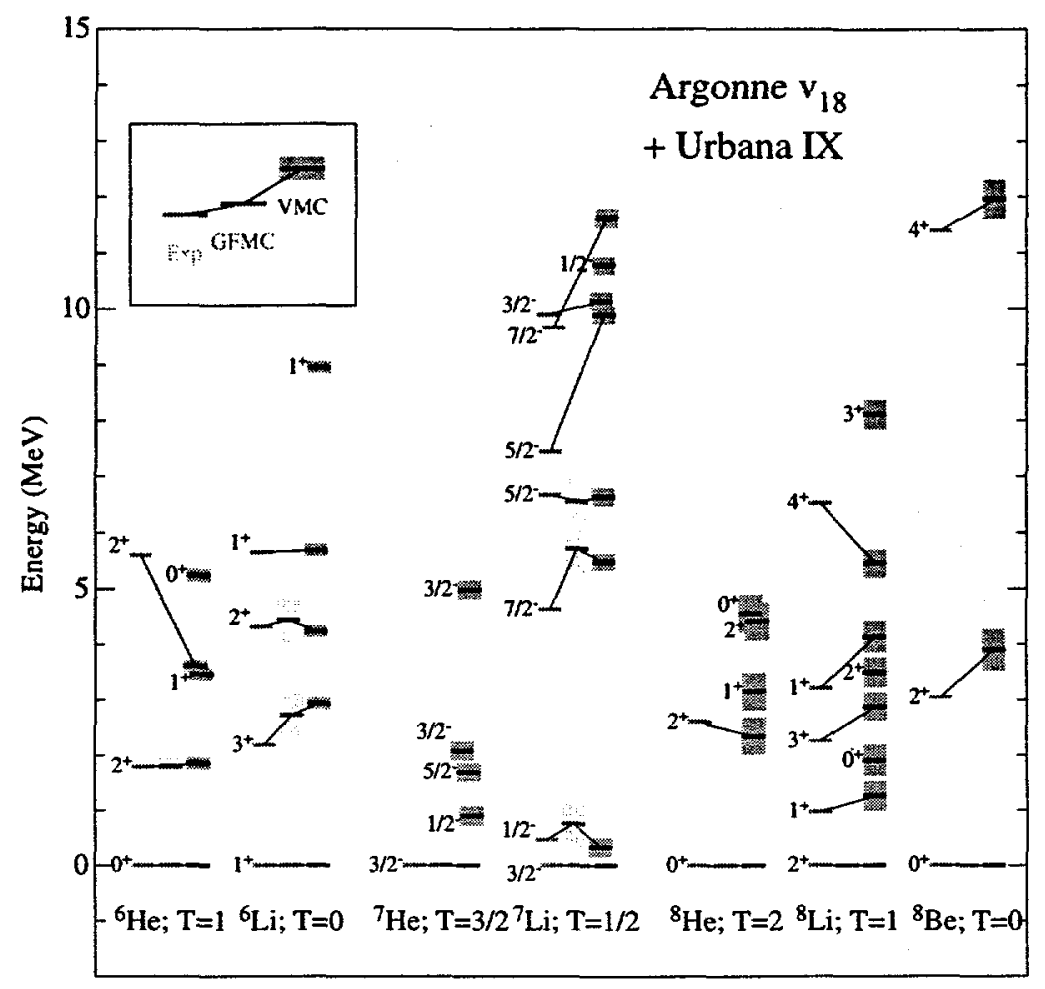

Fig. 3. Experimental and calculated excitation spectrum

experiment. Thus we may reasonably hope that improvements in modelling this phenomenology will make it possible to reproduce the observed energies. Finally, we note that the electromagnetic $v_{i j}^{\gamma}$ is dominated by the Coulomb interaction between protons, but about $17 \%(8 \%)$ of its total contribution comes from the magnetic moment and other terms in $\mathrm{He}(\mathrm{Li})$ isotopes.

Despite the difficulties with the ground state energies, the VMC and GFMC calculations give a reasonable description of the excitation spectra for the $A=6-8$ nuclei. This is seen in Fig. 3 , where we have vertically shifted each column from Fig. 2 to line up the ground states, so the excitation energies can be read off. We have calculated six excited states using both VMC and GFMC, and find good agreement between them and with experiment. An additional 26 states have been calculated with VMC alone, making use of the direct diagonalizations to predict multiple states with the same quantum numbers. In particular, the first excited $\left(1^{+} ; 0\right)$ state in ${ }^{6} \mathrm{Li}$ and the first excited $\left(\frac{3}{2}^{-} ; \frac{1}{2}\right)$ state in ${ }^{7} \mathrm{Li}$ are very close to their experimental values. We also predict a number of states in ${ }^{6} \mathrm{He}$ and ${ }^{7} \mathrm{He}$ which have not been observed. While we generally have the correct ordering of states, the spin-orbit split- 
Table 1. VMC values for proton rms radii (in fm), for magnetic moments (in $\boldsymbol{\mu}_{N}$ ), and quadrupole moments (in $\mathrm{fm}^{2}$ ) in impulse approximation.

\begin{tabular}{|l|l|l|l|l|l|l|}
\hline & \multicolumn{2}{|c|}{$\left\langle r_{p}^{2}\right\rangle^{1 / 2}$} & \multicolumn{2}{c|}{$\mu$} & \multicolumn{2}{c|}{$Q$} \\
\cline { 2 - 6 } & VMC & Expt. & VMC & Expt. & VMC & Expt. \\
\hline${ }^{2} \mathrm{H}\left(1^{+} ; 0\right)$ & 1.967 & 1.975 & 0.847 & 0.857 & 0.270 & 0.286 \\
${ }^{3} \mathrm{H}\left(\frac{1}{2}^{+} ; \frac{1}{2}\right)$ & $1.59(1)$ & 1.60 & $2.582(1)$ & 2.979 & & \\
${ }^{3} \mathrm{He}\left(\frac{1}{2}^{+} ; \frac{1}{2}\right)$ & $1.74(1)$ & 1.77 & $-1.770(1)$ & -2.128 & & \\
${ }^{4} \mathrm{He}\left(0^{+} ; 0\right)$ & $1.47(1)$ & 1.47 & & & & \\
${ }^{6} \mathrm{He}\left(0^{+} ; 1\right)$ & $1.95(1)$ & & & & & \\
${ }^{6} \mathrm{Li}\left(1^{+} ; 0\right)$ & $2.46(2)$ & 2.43 & $0.828(1)$ & 0.822 & $-0.33(18)$ & -0.083 \\
${ }^{6} \mathrm{Be}\left(0^{+} ; 1\right)$ & $2.96(4)$ & & & & & \\
${ }^{7} \mathrm{Li}\left(\frac{3}{2}{ }^{-} ; \frac{1}{2}\right)$ & $2.26(1)$ & 2.27 & $2.924(2)$ & 3.256 & $-3.31(29)$ & -4.06 \\
${ }^{7} \mathrm{Be}\left(\frac{3}{2}-\frac{1}{2}\right)$ & $2.42(1)$ & & $-1.110(2)$ & -1.398 & $-5.64(45)$ & \\
${ }^{8} \mathrm{He}\left(0^{+} ; 2\right)$ & $1.93(2)$ & & & & & \\
${ }^{8} \mathrm{Li}\left(2^{+} ; 1\right)$ & $2.31(2)$ & & $0.976(5)$ & 1.653 & $3.32(37)$ & $3.11(5)$ \\
${ }^{8} \mathrm{Be}\left(0^{+} ; 0\right)$ & $2.29(2)$ & & & & & \\
${ }^{8} \mathrm{~B}\left(2^{+} ; 1\right)$ & $2.66(3)$ & & $1.672(6)$ & 1.036 & $7.08(71)$ & $6.83(21)$ \\
${ }^{8} \mathrm{C}\left(0^{+} ; 2\right)$ & $3.12(5)$ & & & & & \\
\hline
\end{tabular}

tings within multiplets is often too small; for example, the weak splitting of the ${ }^{3} \mathrm{D}_{1,2,3}$ multiplet in ${ }^{6} \mathrm{Li}$ results in a $\left(3^{+} ; 0\right)$ energy that is too high with respect to the ground state.

We have also calculated all the states in $\mathrm{Be}, \mathrm{B}$, and $\mathrm{C}$ that are isobaric analogs to those discussed above using VMC, and some with GFMC. Energy differences among these states are sensitive probes of the chargeindependence-breaking parts of the Hamiltonian. The ${ }^{3} \mathrm{H}-{ }^{3} \mathrm{He}$ mass difference is $760 \mathrm{keV}$ with the present Hamiltonian, in excellent agreement with the experimental value of $764 \mathrm{keV}$. The bulk of the difference is the $p p$ Coulomb energy, but $110 \mathrm{keV}$ comes from the other electromagnetic interactions, the strong interaction CSB term, and the proton-neutron mass difference contribution to the kinetic energy. Energy differences in the p-shell nuclei are not reproduced as well; this could be partially due to the incorrect binding energies of the p-shell nuclei, but it may also reflect the greater uncertainty in the $\mathrm{P}$-wave parts of the $N N$ CIB forces. For example, the experimental ${ }^{7} \mathrm{Be}-{ }^{7} \mathrm{Li}$ mass difference is $1.644 \mathrm{MeV}$, while the theoretical value is only $1.605 \mathrm{MeV}$, with $0.15 \mathrm{MeV}$ coming from the non-Coulomb terms. The CD interactions can also be studied by looking at isotensor energy differences, as in the three $A=6, T=1$ nuclear states [4]. 


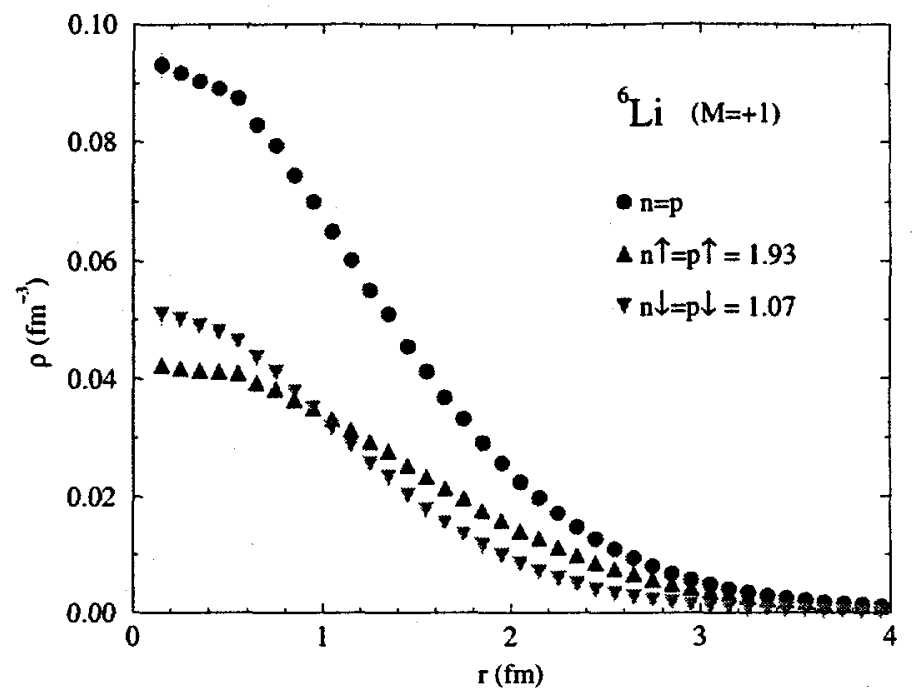

Fig. 4. Spin polarization densities for ${ }^{6} \mathrm{Li}$

\section{Density and Momentum Distributions}

In addition to the energies, we have calculated a variety of one- and twonucleon density distributions, momentum distributions, cluster overlaps, and electromagnetic properties. The proton rms radii and static moments are shown in Table 1. These are calculated from $\Psi_{V}$ using impulse approximation. In general, we know that there can be significant corrections from twobody charge and current contributions [20]. For the magnetic moments, these corrections are only $\sim 1-2 \%$ in isoscalar nuclei like ${ }^{2} \mathrm{H}$, but are $\sim 15-20 \%$ in the isovector $T=\frac{1}{2}$ nuclei ${ }^{3} \mathrm{H}$ and ${ }^{3} \mathrm{He}$. Therefore it is not surprising that we see very little discrepancy for the magnetic moment in ${ }^{6} \mathrm{Li}$, but sizeable errors for ${ }^{7} \mathrm{Li},{ }^{7} \mathrm{Be},{ }^{8} \mathrm{Li}$, and ${ }^{8} \mathrm{~B}$. Presumably, calculations including mesonexchange contributions would come much closer to the experimental values in the latter cases.

The small quadrupole moment of ${ }^{6} \mathrm{Li}$ is a difficult problem, in which there is a delicate cancellation between the contributions from the deuteron quadrupole moment and the $\mathrm{D}$-wave part of the $\alpha-d$ relative wave function. Many cluster models for ${ }^{6} \mathrm{Li}$ fail to obtain the observed negative sign; we have the correct sign but too large a magnitude. In the case of ${ }^{7} \mathrm{Li}$, in which there is no such delicate cancellation, the value is only $\sim 15-20 \%$ too low in magnitude, while the ${ }^{8} \mathrm{Li}$ and ${ }^{8} \mathrm{~B}$ values are surprisingly good.

In Fig. 4 we give the proton and neutron spin densities in ${ }^{6} \mathrm{Li}$, which may be of use in the characterization of spin-polarized ${ }^{6} \mathrm{LiD}$ as an effective polarized neutron target. The integrated neutron densities are found to be 1.93 

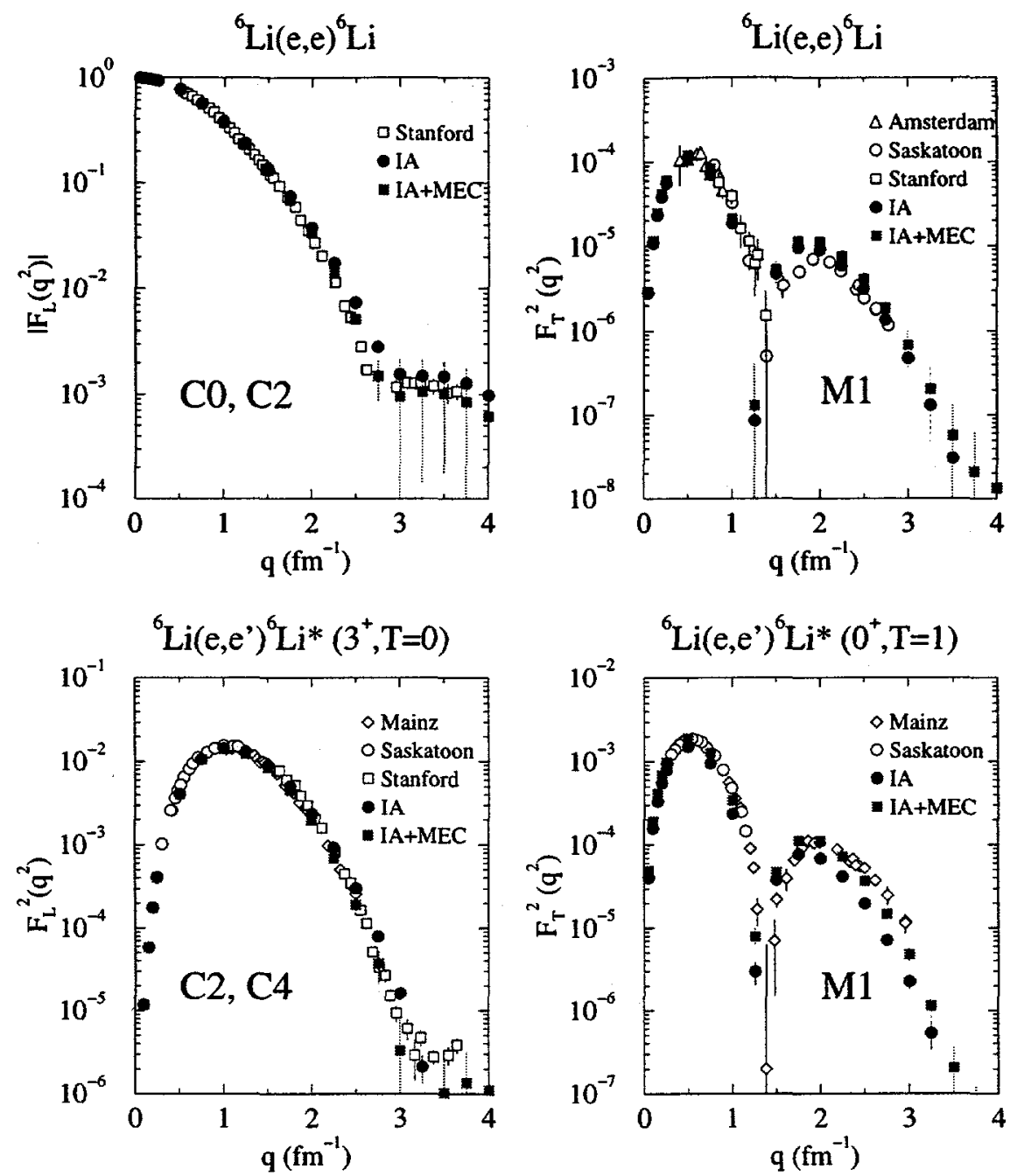

Fig. 5. Calculated elastic and transition form factors in ${ }^{6} \mathrm{Li}$

for spin up and 1.09 for spin down, respectively, yielding a net polarization of $29 \%$ [4].

VMC calculations of elastic and inelastic electromagnetic form factors for ${ }^{6} \mathrm{Li}$ are shown in Fig. 5. These have been made in impulse approximation (IA) and with meson-exchange contributions (MEC) to the charge and density operators [21]. The comparison with data for the elastic longitudinal form factor, $F_{L}\left(q^{2}\right)$, is excellent, as is the $\mathrm{E} 2$ transition to the $3^{+}$first excited state. The MEC corrections are small, but stand out noticeably in the first minimum where they significantly improve the fit to data. The elastic transverse form 


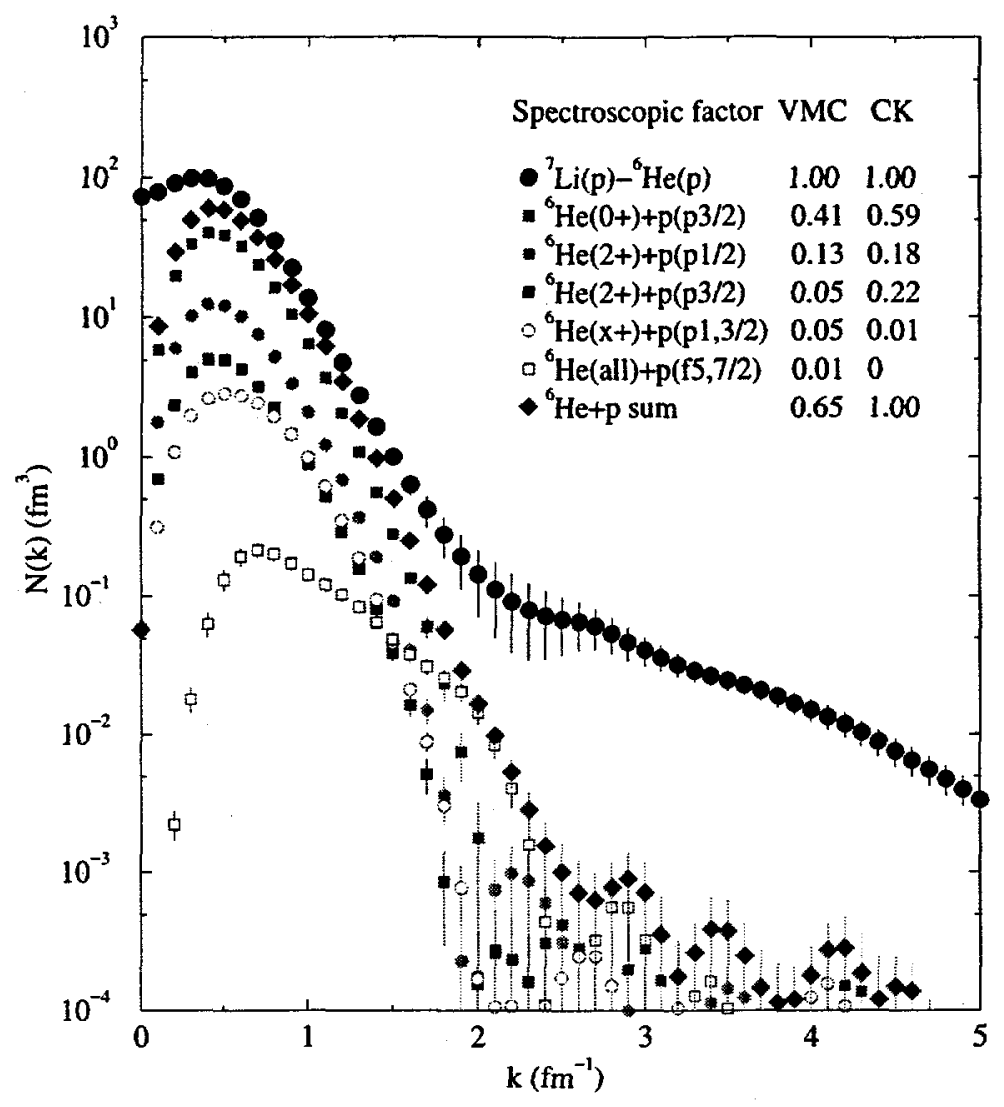

Fig. 6. Momentum distributions and cluster overlaps for $A=6,7$ nuclei

factor, $F_{T}\left(q^{2}\right)$, is good up to the first zero, but is a little too large in the region of the second maximum. The M1 transition to the $0^{+}$isobaric analog state is also reproduced reasonably well.

We have also calculated single-nucleon momentum distributions in these nuclei, and a variety of cluster-cluster overlap wave functions, such as $\langle d p \mid t\rangle$, $\langle d d \mid \alpha\rangle$, and $\left\langle\left.\alpha d\right|^{6} \mathrm{Li}\right\rangle[22,23]$. Recently we have studied the overlaps $\left({ }^{6} \mathrm{He}\left(J^{\pi}\right)+\right.$ $\left.\left.p\left(\ell_{j}\right)\right|^{7} \mathrm{Li}\right\rangle$ shown in Fig. 6 , for $J=0,2$, or higher, $\ell=$ p or $\mathrm{f}$, and $j=\frac{1}{2}$ to $\frac{7}{2}$. They are compared to the difference of the single-proton momentum distributions in ${ }^{7} \mathrm{Li}$ and ${ }^{6} \mathrm{He}$, which should approximate the one p-shell proton wave function. The spectroscopic factors obtained from these overlaps are given in the figure and compared to the predictions of the Cohen-Kurath shell model [15]. There is a noticeable quenching of the strength in the present analysis compared to traditional shell model, as has been observed in ${ }^{7} \mathrm{Li}\left(e, e^{\prime} p\right)^{6} \mathrm{He}$ experiments [24]. 


\section{Conclusions and Outlook}

Quantum Monte Carlo methods can now be used to obtain accurate (within $1 \%$ ) energies for light $p$-shell nuclei up to $A=8$. This has been made possible by the rapid growth of computational power and a continuing evolution of algorithms, often inspired by work in condensed matter physics. With these calculations, we can see for the first time the nuclear shell structure emerging directly from bare $N N$ interactions that fit two-body scattering data.

The future of QMC calculations in light nuclei is very promising. A new algorithm, called 'constrained path' GFMC [25], promises a significant reduction in the fermion sign noise and the consequent ability to propagate to larger Euclidean time $\tau$. The present method is being extended to slightly larger nuclei, where we expect ${ }^{9} \mathrm{Be}\left({ }^{10} \mathrm{~B}\right)$ to be about 4 (12) times more expensive to compute than ${ }^{8} \mathrm{Li}$. For larger nuclei, we are working on a fusion of the cluster VMC methods [26] that have been used in ${ }^{16} \mathrm{O}$, with the GFMC algorithm.

A major effort is now underway to improve the Hamiltonian, particularly the $V_{i j k}^{R}$, to fit the observed $A=6-8$ spectrum at the $1 \%$ level. Additions include the relativistic boost correction $[10,11]$ that is about $\sim 35 \%$ of $V_{i j k}^{R}$ in ${ }^{3} \mathrm{H}$ and ${ }^{4} \mathrm{He}$. We are also examining three-pion-exchange and spin-orbit three-nucleon forces, which are small in magnitude, but may significantly improve the overall spectrum.

In addition there are many astrophysical applications for the $A=6-8$ nuclear wave functions, such as the radiative captures ${ }^{4} \mathrm{He}(d, \gamma){ }^{6} \mathrm{Li}$, and ${ }^{4} \mathrm{He}(t, \gamma){ }^{7} \mathrm{Li}$ for primordial nucleosynthesis, and ${ }^{7} \mathrm{Be}(p, \gamma)^{8} \mathrm{~B}$ for the solar neutrino problem. QMC methods are also being used to study neutron 'drops', finite systems of neutrons in an external potential well [27], with applications in neutron-rich nuclei like those found in the crusts of neutron stars.

\section{Acknowledgments}

It is a pleasure to thank my colleagues A. Arriaga, J. Carlson, J.L. Forest, V.R. Pandharipande, S.C. Pieper, B.S. Pudliner, R. Schiavilla, and V.G.J. Stoks for their contributions to this work. The calculations described here were made possible by generous grants of time from the Mathematical and Computer Science Division of Argonne National Laboratory, and the Cornell Theory Center. This work is supported by the U.S. Department of Energy, Nuclear Physics Division, under Contract No. W-31-109-ENG-38.

\section{References}

1. R.B. Wiringa in Contemporary Nuclear Shell Models, ed. X.-W. Pan, D.H. Feng, and M. Vallières (Springer-Verlag, Berlin, 1997)

2. J. Carlson and R. Schiavilla, Rev. Mod. Phys. 70, 743 (1998) 
3. B.S. Pudliner, V.R. Pandharipande, J. Carlson, and R.B. Wiringa, Phys. Rev. Lett. 74, 4396 (1995)

4. B.S. Pudliner, V.R. Pandharipande, J. Carlson, S.C. Pieper, and R.B. Wiringa, Phys. Rev. C 56, 1720 (1997)

5. R.B. Wiringa, V.G.J. Stoks, and R. Schiavilla, Phys. Rev. C 51, 38 (1995)

6. J.R. Bergervoet, P.C. van Campen, R.A.M. Klomp, J.-L. de Kok, T.A. Rijken, V.G.J. Stoks, and J.J. de Swart, Phys. Rev. C 41, 1435 (1990)

7. V.G.J. Stoks, R.A.M. Klomp, M.C.M. Rentmeester, and J.J. de Swart, Phys. Rev. C 48, 792 (1993)

8. J. Fujita and H. Miyazawa, Prog. Theor. Phys. 17, 360 (1957)

9. S.A. Coon, M.T. Peña, and D.O. Riska, Phys. Rev. C 52, 2925 (1995)

10. J.L. Forest, V.R. Pandharipande, and J.L. Friar, Phys. Rev. C 52, 568 (1995)

11. J.L. Forest, V.R. Pandharipande, J. Carlson, and R. Schiavilla, Phys. Rev. C 52, 576 (1995)

12. R.B. Wiringa, Rev. Mod. Phys. 65, 231 (1993)

13. A. Arriaga, V.R. Pandharipande, and R.B. Wiringa, Phys. Rev. C 52, 2362 (1995)

14. R.B. Wiringa, Phys. Rev. C 43, 1585 (1991)

15. S. Cohen and D. Kurath, Nucl. Phys. 73, 1 (1965); Nucl. Phys. A101, 1 (1967)

16. A. Bohr and B.R. Mottelson, Nuclear Structure Volume I, (W. A. Benjamin, New York, 1969)

17. J.A. Carlson and R.B. Wiringa, in Computational Nuclear Physics 1, ed. K. Langanke, J. A. Maruhn, and S. E. Koonin (Springer Verlag, Berlin, 1991)

18. J. Carlson, Phys. Rev. C 36, 2026 (1987) Phys. Rev. C 38, 1879 (1988)

19. D.M. Ceperley and M.H. Kalos, in Monte Carlo Methods in Statistical Physics, ed. K. Binder (Springer-Verlag, Heidelberg, 1979)

20. R. Schiavilla, V.R. Pandharipande, and D.O. Riska, Phys. Rev. C 40, 2294 (1989); Phys. Rev. C 41, 309 (1990)

21. R.B. Wiringa and R. Schiavilla, Phys. Rev. Lett., to be published (1998)

22. R. Schiavilla, V.R. Pandharipande, and R.B. Wiringa, Nucl. Phys. A449, 219 (1986)

23. J.L. Forest, V.R. Pandharipande, S.C. Pieper, R.B. Wiringa, R. Schiavilla, and A. Arriaga, Phys. Rev. C 54, 646 (1996)

24. L. Lapikás, J. Wesseling, and R.B. Wiringa, submitted to Phys. Rev. Lett.

25. S. Zhang, J. Carlson, and J.E. Gubernatis, Phys. Rev. Lett. 74, 3653 (1995)

26. S.C. Pieper, R.B. Wiringa, and V.R. Pandharipande, Phys. Rev. C 46, 1741 (1992)

27. B.S. Pudliner, A. Smerzi, J. Carlson, V.R. Pandharipande, S.C. Pieper, and D.G. Ravenhall, Phys. Rev. Lett. 76, 2416 (1996) 